\title{
Ekeland Variational Principle for Generalized Vector Equilibrium Problems with Equivalences and Applications
}

\author{
De-ning $Q u^{1,2}$ and Cao-zong Cheng \\ ${ }^{1}$ College of Applied Science, Beijing University of Technology, Beijing 100124, China \\ ${ }^{2}$ College of Mathematics, Jilin Normal University, Siping, Jilin 136000, China \\ Correspondence should be addressed to De-ning Qu; qudening@126.com
}

Received 2 April 2013; Accepted 12 July 2013

Academic Editor: Yongsheng S. Han

Copyright (C) 2013 D.-n. Qu and C.-z. Cheng. This is an open access article distributed under the Creative Commons Attribution License, which permits unrestricted use, distribution, and reproduction in any medium, provided the original work is properly cited.

The aim of this paper is to introduce Ekeland variational principle with variants for generalized vector equilibrium problems and to establish some existence results of solutions of generalized vector equilibrium problems with compact or noncompact domain as applications. Finally, some equivalent results of the established Ekeland variational principle are presented.

\section{Introduction}

An Ekeland variational principle [1] (also [2, 3]) appeared first as an existence result of approximate minimizer for a lower semicontinuous and bounded below function on complete metric spaces. It subsequently developed an important tool of many subjects, such as in nonlinear analysis (e.g., [4]), optimization theory (e.g., [5-11]), game theory (e.g., [12]), dynamical systems (e.g., [13]), and others (e.g., [14, 15]). By reason of the fact that equilibrium problems contain many problems as their special cases, such as optimization problems, fixed-point problems, variational inequality problems, complementary problems, and Nash equilibrium problems (see [16]), a new direction of research on variational principle for equilibrium problems has arisen. The variational principle for equilibrium problems (e.g., [17]) and for vector equilibrium problems (e.g., [18-23]) and/or their applications or equivalent results were discussed. In terms of set-valued objective mappings, variational principle for vector optimization problems was first introduced by Chen and Huang [5] and was reported in many literatures (e.g., see [7, 9-11]) in the sequel. In 2009, Zeng and Li [24] discussed Ekeland variational principle for vector equilibrium problems with setvalued objective mappings (generalized vector equilibrium problems).
One of the most important tools of obtaining variational principle for vector problems is the scalarization functions (see $[5,6,21-24]$, for instance). While these scalarization functions always involve single-valued mappings. Motivated by the works mentioned above, we establish the Ekeland variational principle for generalized vector equilibrium problems by applying a nonlinear scalarization function involving set-valued mappings. It is worth noting that the generalized vector equilibrium problems considered in this paper are rather than those in [24].

Let $\mathbb{R}, \mathbb{R}_{+}$and $\mathbb{N}$ be denoted by the sets of real numbers, nonnegative real numbers and positive integers, respectively, and let $\mathcal{N}(*)$ be the collection of open neighborhoods of $*$, where $*$ is a point or a set. A subset $D$ of a real topological vector space $Y$ is called a cone if $\lambda x \in D$ for all $x \in D$ and $\lambda>0$. Let $D$ be a cone in $Y$ and $A \subset Y$. D is called proper if $D \neq Y$. $A$ is said to be $D$-closed [25] if $A+\operatorname{cl} D$ is closed; to be $D$-bounded [25] if, for each neighborhood $U \in \mathcal{N}\left(0_{Y}\right)$, there exists $\lambda>0$ such that $A \subset \lambda U+D$.

Throughout this paper, unless otherwise specified, let $X$ be a Hausdorff topological space and $Y$ a real Hausdorff topological vector space, let $D \subset Y$ be a proper, closed, and convex cone with nonempty interior and $e \in \operatorname{int} D$, and let $f: X \times X \rightarrow 2^{Y}$ be a strict, set-valued mapping, 
$f_{x}(\cdot)=f(x, \cdot)$ for each $x \in X$ and $f^{y}(\cdot)=f(\cdot, y)$ for each $y \in X$. A set-valued mapping is said to be strict if it has nonempty values. Consider the following generalized vector equilibrium problems:

$$
\begin{aligned}
& \text { to find } \bar{x} \in X \text { such that } f(\bar{x}, y) \cap(-D)=\emptyset \text {, } \\
& \forall y \in X \text {, }
\end{aligned}
$$

to find $\bar{x} \in X$ such that $f(\bar{x}, y) \cap(-\operatorname{int} D)=\emptyset$,

$$
\forall y \in X \text {. }
$$

This paper is divided into five sections. In Section 2, some preliminaries are provided. In Section 3, Ekeland variational principle with variants for (GVEP1) is argued in complete quasimetric spaces. In Section 4, some existence results of solutions of (GVEP2) with compact or noncompact domain are established as applications. Finally, in Section 5, some equivalent results of the established Ekeland variational principle are presented.

\section{Preliminaries}

Let $X$ be a nonempty set. “ $\preceq$ ” is called a quasiorder on $X$ if it is of

(o1) reflexivity: $x \preceq x$, for all $x \in X$;

(o2) transitivity: $x \preceq y, y \preceq z \Rightarrow x \preceq z$.

Here $(X, \preceq)$ is called a quasiorder space. An element $x \in X$ is said to be a maximal element of a quasiorder space $(X, \preceq)$ if there is no element $y \in X$, other than $x$, such that $x \preceq y$, in other words, $x \preceq y$ for some $y \in X$ implies $x=y$.

Let $X$ and $Y$ be topological spaces. A real-valued function $g: X \rightarrow \mathbb{R}$ is said to be upper semicontinuous on $X$ if $\{u \in X: g(u)<\lambda\}$ is open for each $\lambda \in \mathbb{R}$; to be lower semicontinuous on $X,\{u \in X: g(u)>\lambda\}$ is open for each $\lambda \in \mathbb{R}$. The following conceptions of continuity for a setvalued mapping can be found in [4]. A set-valued mapping $G: X \rightarrow 2^{Y}$ is said to be upper semicontinuous at $u_{0} \in X$ if, for any $N \in \mathcal{N}\left(G\left(u_{0}\right)\right)$, there exists $B \in \mathcal{N}\left(u_{0}\right)$ such that $G(u) \subset N$ for all $u \in B$; to be lower semicontinuous at $u_{0} \in X$, if for any $y_{0} \in G\left(u_{0}\right)$ and any $N \in \mathcal{N}\left(y_{0}\right)$, there exists $B \in \mathcal{N}\left(u_{0}\right)$ such that $G(u) \cap N \neq \emptyset$ for all $u \in B$; to be upper semicontinuous (resp., lower semicontinuous) on $X$, if $G$ is upper semicontinuous (resp., lower semicontinuous) at each $u \in X$; to be continuous at $u_{0} \in X$ (resp., on $X$ ), if $G$ is both upper semicontinuous and lower semicontinuous at $u_{0}$ (resp., on $X)$; to be closed, if its graph $\operatorname{Graph}(G)=\{(u, y) \in$ $X \times Y: y \in G(u)\}$ is closed in $X \times Y$.

Lemma 1 (see [26]). Let $X$ and $Y$ be Hausdorff topological spaces and $S: X \rightarrow 2^{Y}$ a strict set-valued mapping. If $X$ is compact and $S$ is upper semicontinuous with compact values, then $S(X)=\cup\{S(x): x \in X\}$ is compact.
Definition 2. Let $X$ be a nonempty set. A function $d: X \times$ $X \rightarrow \mathbb{R}_{+}$is said to be a quasimetric on $X$ if

(d1) $d(x, y)=0$ if and only if $x=y$;

(d2) $d(x, z) \leq d(x, y)+d(y, z), \forall x, y, z \in X$.

$X$ equipped a quasimetric $d$ is said to be a quasimetric space, denoted by $(X, d)$. Furthermore, if $d$ also satisfies

(d3) $d(x, y)=d(y, x), \forall x, y \in X$,

then $(X, d)$ is called a metric space, where $d$ is a metric on $X$.

Let $(X, d)$ be a quasimetric space. $x_{k} \rightarrow x$ means $d\left(x_{k}, x\right) \rightarrow 0 .\left\{x_{k}\right\} \subset X$ is called a Cauchy sequence on $(X, d)$ if, for any $\varepsilon>0$, there exists $K \in \mathbb{N}$ such that $d\left(x_{k}, x_{l}\right)<$ $\varepsilon, \forall l>k>K .(X, d)$ is said to be complete if, for each Cauchy sequence $\left\{x_{k}\right\} \subset X$, there exists $x \in X$ such that $x_{k} \rightarrow x$ as $k \rightarrow+\infty$.

Definition 3 (see [27]). Let $(X, d)$ be a quasimetric space. A function $w: X \times X \rightarrow \mathbb{R}_{+}$is said to be a $W$-distance on $(X, d)$ if

(w1) $w(x, z) \leq w(x, y)+w(y, z), \forall x, y, z \in X$;

(w2) for each fixed $x \in X, y \mapsto w(x, y)$ is lower semicontinuous;

(w3) for any $\epsilon>0$, there exists $\delta>0$ such that $w(x, y) \leq \delta$ and $w(x, z) \leq \delta$ imply that $d(y, z) \leq \epsilon$.

The $W$-distance includes metric and quasimetric as its special cases. But the converse fails to be true. Moreover, $W$ distance is not necessary to be symmetric. Some examples and properties of a $W$-distance in metric spaces are provided by Kada et al. [27].

Lemma 4 (see [27]). Let $w$ be a $W$-distance on a quasimetric space $(X, d)$, let $\left\{x_{k}\right\},\left\{y_{k}\right\} \subset X$ and $\left\{\alpha_{k}\right\},\left\{\beta_{k}\right\} \subset \mathbb{R}_{+}$with $\alpha_{k}, \beta_{k} \rightarrow 0$ as $k \rightarrow+\infty$, and let $x, y, z \in X$. Then the following assertions are true:

(i) if $w\left(x_{k}, y\right) \leq \alpha_{k}$ and $w\left(x_{k}, z\right) \leq \beta_{k}, \forall k \in \mathbb{N}$, then $y=z$. In particular, if $w(x, y)=0$ and $w(x, z)=0$, then $y=z$;

(ii) if $w\left(x_{k}, x_{l}\right) \leq \alpha_{k}, \forall l>k$, then $\left\{x_{k}\right\}$ is a Cauchy sequence;

(iii) ift $>0$, then tw is also a $W$-distance on $(X, d)$.

Let $X$ be a topological space and $Y$ be a topological vector space in the rest of this section.

Since each compact subset in $Y$ is both $D$-closed and D-bounded by Definition 3.1 and Proposition 3.1 in [25], the following general nonlinear scalarization function is well defined in view of Lemma 3.1 in [28].

Definition 5. Let $G: X \rightarrow 2^{Y}$ be a strict and compact-valued mapping. A generalized nonlinear scalarization function $\zeta_{G}$ : $X \rightarrow \mathbb{R}$ of $G$ is defined by

$$
\begin{array}{r}
\zeta_{G}(u)=\min \{\lambda \in \mathbb{R}: G(u) \cap(\lambda e-D) \neq \emptyset\}, \\
\forall u \in X .
\end{array}
$$



ing.

According to Proposition 3.1 in [28], we have the follow-

Lemma 6. Let $G: X \rightarrow 2^{Y}$ be a strict and compact-valued mapping. The following assertions are true for each $\lambda \in \mathbb{R}$ and $u \in X$ :

(i) $\zeta_{G}(u)<\lambda \Leftrightarrow G(u) \cap(\lambda e-\operatorname{int} D) \neq \emptyset$.

(ii) $\zeta_{G}(u) \leq \lambda \Leftrightarrow G(u) \cap(\lambda e-D) \neq \emptyset$.

Lemma 7. Let $G: X \rightarrow 2^{Y}$ be a strict and compact-valued mapping.

(i) If $G$ is lower semicontinuous on $X$, then $\zeta_{G}$ is upper semicontinuous on $X$;

(ii) If $G$ is upper semicontinuous on $X$, then $\zeta_{G}$ is lower semicontinuous on $X$.

Proof. This proof is completed by letting $G(x)=0, C(x)=$ $D$, and $E(x)=\{e\}$ for all $x \in X$ in Corollary 3.1 in [28] since upper (resp., lower) semicontinuity implies the $D$-upper (resp., lower) semicontinuity (see [29]).

Lemma 8. If

(a1) $f$ is compact valued;

(a2) $f(x, y)+f(y, z) \subset f(x, z)+D, \forall x, y, z \in X$,

then

$$
\zeta_{f_{x}}(z) \leq \zeta_{f_{x}}(y)+\zeta_{f_{y}}(z), \quad \forall x, y, z \in X
$$

Proof. For each $x, y, z \in X$, it follows from Definition 5 that $u \in f(x, y) \cap\left(\zeta_{f_{x}}(y) e-D\right)$ and $v \in f(y, z) \cap\left(\zeta_{f_{y}}(z) e-D\right)$ can be chosen. Then $u+v \in f(x, z)+D$ by (a2). Select $w \in f(x, z)$ to satisfy $w \in u+v-D$. This deduces that

$$
\begin{array}{r}
w \in\left(\zeta_{f_{x}}(y) e-D\right)+\left(\zeta_{f_{y}}(z) e-D\right) \\
-D \subset\left(\zeta_{f_{x}}(y)+\zeta_{f_{y}}(z)\right) e-D .
\end{array}
$$

Therefore, $f(x, z) \cap\left(\left(\zeta_{f_{x}}(y)+\zeta_{f_{y}}(z)\right) e-D\right) \neq \emptyset$ by Lemma 6(ii) and $\zeta_{f_{x}}(z) \leq \zeta_{f_{x}}(y)+\zeta_{f_{y}}(z)$.

\section{Ekeland Variational Principle for (GVEP1)}

From now on, unless otherwise specified, suppose that $(X, d)$ is a Hausdorff complete quasimetric space and that $w$ is a $W$ distance on $(X, d)$.

Lemma 9. (i) If (a1)-(a2) and

(a3) $f(x, x) \cap(-\operatorname{int} D)=\emptyset, \forall x \in X$

hold, then

$$
S(y) \subset S(x), \quad \forall y \in S(x), \forall x \in X,
$$

where $S: X \rightarrow 2^{X}$ is defined by

$$
\begin{array}{r}
S(x)=\{y \in X: y \neq x, f(x, y) \cap(-w(x, y) e-D) \neq \emptyset\}, \\
\forall x \in X .
\end{array}
$$

(ii) Besides (a1)-(a3), if

(a4) for each $x \in X, f_{x}(\cdot)$ is upper semicontinuous on $X$;

(a5) for each $x \in X, f_{x}(X) \cap(b-D)=\emptyset$ for some $b \in Y$,

then, for each $\widehat{x} \in X$ with $S(\widehat{x}) \neq \emptyset$, there exists $\bar{x} \in S(\widehat{x})$ such that $S(\bar{x})=\emptyset$.

Proof. Obviously, by (a1) and Lemma 6(ii),

$$
S(x)=\left\{y \in X: y \neq x, \zeta_{f_{x}}(y)+w(x, y) \leq 0\right\} .
$$

(i) For each $x \in X$ and $y \in S(x)$, if $S(y)=\emptyset$, then the conclusion holds trivially. Otherwise, for each $z \in S(y)$,

$$
\zeta_{f_{x}}(y)+w(x, y) \leq 0, \quad \zeta_{f_{y}}(z)+w(y, z) \leq 0,
$$

and so

$$
\zeta_{f_{x}}(z)+w(x, z) \leq \zeta_{f_{x}}(y)+\zeta_{f_{y}}(z)+w(x, y)+w(y, z) \leq 0
$$

according to Lemma 8 . Moreover, by $w(x, y) \geq 0$ and $w(y, z) \geq 0$,

$$
\zeta_{f_{x}}(y) \leq 0, \quad \zeta_{f_{y}}(z) \leq 0
$$

Now claim that $z \neq x$. Otherwise, $\zeta_{f_{x}}(z) \geq 0$ by (a3) and Lemma 6(i). Thus,

$$
w(x, y)=0
$$

since

$$
\begin{aligned}
w(x, y) & \leq \zeta_{f_{x}}(z)+w(x, y) \\
& \leq \zeta_{f_{x}}(y)+w(x, y)+\zeta_{f_{y}}(z) \\
& \leq 0
\end{aligned}
$$

by (2) and (9). Similarly, $w(y, z)=0$, and so $w(x, z)=0$ by (w1). This, together with $w(x, y)=0$, implies that $y=z$ by Lemma 4(i), which contradicts with $z \in S(y)$. Thus $z \in S(x)$, and so $S(y) \subset S(x), \forall y \in S(x)$.

(ii) For $b \in Y$ given in (a5), $b \in r e+D$ for some $r \in \mathbb{R}$ by Lemma 2.1 in [30], which leads to $r e-D \subset b-D$. This, together with (a5), implies that $f_{x}(X) \cap(r e-D)=\emptyset$. It follows form Lemma 4(ii) that

$$
v(x)=\inf \left\{\zeta_{f_{x}}(y): y \in S(x)\right\}>-\infty, \quad \forall x \in X .
$$

Then, for each $x_{0}=\widehat{x} \in X$,

$$
v\left(x_{0}\right)=\inf \left\{\zeta_{f_{x_{0}}}(y): y \in S\left(x_{0}\right)\right\}>-\infty .
$$


For each $k \in \mathbb{N}$, let $x_{k-1}$ satisfy that $v\left(x_{k-1}\right)>-\infty$ and take $x_{k} \in S\left(x_{k-1}\right)$ such that

$$
\zeta_{f_{x_{k-1}}}\left(x_{k}\right) \leq v\left(x_{k-1}\right)+\frac{1}{2^{k}}
$$

If $S\left(x_{k}\right)=\emptyset$ for some $k \in \mathbb{N}$, this conclusion holds by letting $\bar{x}=x_{k}$. Now consider $S\left(x_{k}\right) \neq \emptyset, \forall k \in \mathbb{N}$. The rest proof is divided into four steps as follows.

(a) Show that $x_{k} \rightarrow \bar{x}$ as $k \rightarrow+\infty$ for some $\bar{x} \in X$. Indeed, for each $k \in \mathbb{N}$, by (2),

$$
\begin{aligned}
v\left(x_{k}\right) & =\inf \left\{\zeta_{f_{x_{k}}}(y): y \in S\left(x_{k}\right)\right\} \\
& \geq \inf \left\{\zeta_{f_{x_{k-1}}}(y)-\zeta_{f_{x_{k-1}}}\left(x_{k}\right): y \in S\left(x_{k-1}\right)\right\} \\
& =v\left(x_{k-1}\right)-\zeta_{f_{x_{k-1}}}\left(x_{k}\right) \geq-\frac{1}{2^{k}}
\end{aligned}
$$

which implies that

$$
w\left(x_{k}, y\right) \leq-\zeta_{f_{x_{k}}}(y) \leq-v\left(x_{k}\right) \leq \frac{1}{2^{k}}, \quad \forall y \in S\left(x_{k}\right),
$$

and so

$$
w\left(x_{k}, x_{l}\right) \leq \sum_{i=k}^{l-1} w\left(x_{i}, x_{i+1}\right) \leq \sum_{i=k}^{l-1} \frac{1}{2^{i}} \leq \frac{1}{2^{k-1}}, \quad \forall l>k .
$$

Therefore, $\left\{x_{k}\right\}$ is a Cauchy sequence by Lemma 4(ii), and so there exists $\bar{x} \in X$ such that $x_{k} \rightarrow \bar{x}$ as $k \rightarrow+\infty$ by the completeness of $(X, d)$. In addition, according to (w2)

$$
w\left(x_{k}, \bar{x}\right) \leq \liminf _{l \rightarrow+\infty} w\left(x_{k}, x_{l}\right) \leq \frac{1}{2^{k-1}}, \quad \forall k \in \mathbb{N}
$$

(b) Show that $\bar{x} \in \bigcap_{k=0}^{+\infty} S\left(x_{k}\right)$. In fact, for each $k \in \mathbb{N}$,

$$
\zeta_{f_{x_{k}}}\left(x_{l}\right)+w\left(x_{k}, x_{l}\right) \leq \sum_{i=k}^{l-1}\left(\zeta_{f_{x_{i}}}\left(x_{i+1}\right)+w\left(x_{i}, x_{i+1}\right)\right) \leq 0
$$

by (2). Since, for each $x \in X, \zeta_{f_{x}}(\cdot)$ is lower semicontinuous by (a1), (a4), and Lemma 7(ii),

$$
\zeta_{f_{x_{k}}}(\bar{x})+w\left(x_{k}, \bar{x}\right) \leq 0
$$

by letting $l \rightarrow+\infty$ in (19). Hence,

$$
\zeta_{f_{x_{k}}}(\bar{x}) \leq 0, \quad \forall k \in \mathbb{N}
$$

Now it is enough to prove that $x_{k} \neq \bar{x}, \forall k \in \mathbb{N}$ by (20). Indeed, if $x_{k_{0}}=\bar{x}$ for some $k_{0} \in \mathbb{N}$, then $\zeta_{f_{x_{0}}}\left(x_{k_{0}}\right)=\zeta_{f_{x_{0}}}(\bar{x}) \geq 0$ in view of (a3) and Lemma 6(i). By applying (21) and adopting the same argument of the proof of (10),

$$
w\left(x_{k_{0}}, x_{k_{0}+1}\right)=0 \text {. }
$$

Thus,

$$
\zeta_{f_{x_{0}}}\left(x_{k_{0}+1}\right)=\zeta_{f_{x_{k_{0}}}}\left(x_{k_{0}+1}\right)+w\left(x_{k_{0}}, x_{k_{0}+1}\right) \leq 0 .
$$

It follows form $0 \leq \zeta_{f_{x_{k_{0}}}}(\bar{x}) \leq \zeta_{f_{x_{k_{0}}}}\left(x_{k_{0}+1}\right)+\zeta_{f_{x_{k_{0}+1}}}(\bar{x})$ that $\zeta_{f_{x_{0}+1}}(\bar{x}) \geq-\zeta_{f_{x_{0}}}\left(x_{k_{0}+1}\right) \geq 0$. Hence, $w\left(x_{k_{0}+1}, x_{k_{0}+2}\right)=0$ by (21) and the similar argument of (10), which, together with $w\left(x_{k_{0}}, x_{k_{0}+2}\right)=0$, implies $x_{k_{0}+1}=x_{k_{0}+2}$ in virtue of (22) and Lemma 4(i). This contradicts with $x_{k_{0}+2} \in S\left(x_{k_{0}+1}\right)$. Thus, $\bar{x} \in$ $S\left(x_{k}\right), \forall k \in \mathbb{N}$. Clearly, $\bar{x} \in S\left(x_{0}\right)$.

(c) Show that $\bigcap_{k=1}^{+\infty} S\left(x_{k}\right)=\{\bar{x}\}$. Indeed, for any $\tilde{x} \in$ $\bigcap_{k=1}^{+\infty} S\left(x_{k}\right), \tilde{x} \neq x_{k}$, and $\zeta_{f_{x_{k}}}(\tilde{x})+w\left(x_{k}, \tilde{x}\right) \leq 0, \forall k \in \mathbb{N}$. As a result,

$$
w\left(x_{k}, \tilde{x}\right) \leq-\zeta_{f_{x_{k}}}(\tilde{x}) \leq-v\left(x_{k}\right) \leq \frac{1}{2^{k}}, \quad \forall k \in \mathbb{N}
$$

which, together with (18), implies that $\tilde{x}=\bar{x}$ by Lemma 4(i).

(d) Show that $\bar{x} \in S(\widehat{x})$ and $S(\bar{x})=\emptyset$. As a matter of fact, $\bar{x} \in S(\widehat{x})$ by (b). If $S(\bar{x}) \neq \emptyset$, then $S(\bar{x})=\{\bar{x}\}$ in view of $(\mathrm{c})$. This leads to a contradiction by the definition of $S$.

Theorem 10. Define $A: X \rightarrow 2^{X}$ as

$$
\begin{array}{r}
A(x) \\
=\{y \in X: x=y \text { or } f(x, y) \cap(-\varepsilon w(x, y) e-D) \neq \emptyset\}, \\
\forall x \in X .
\end{array}
$$

If (a1)-(a5) hold, then, for any $\varepsilon>0$ and for any $\widehat{x} \in X$, there exists $\bar{x} \in X$ such that

(i) $A(\bar{x})=\{\bar{x}\}$;

(ii) $f(\bar{x}, y) \cap(-\varepsilon w(\bar{x}, y) e-D)=\emptyset, \forall y \in X$ with $y \neq \bar{x}$;

(iii) $f(\widehat{x}, \bar{x}) \cap(-\varepsilon w(\widehat{x}, \bar{x}) e-D) \neq \emptyset$ if, further, $S(\widehat{x}) \neq \emptyset$, where $S: X \rightarrow 2^{X}$ defined as

$$
S(x)=\{y \in X: x \neq y, f(x, y) \cap(-\varepsilon w(x, y) e-D) \neq \emptyset\},
$$

Proof. Since $w_{\varepsilon}(x, y)=\varepsilon w(x, y)$ is another $W$-distance by Lemma 4(iii), without loss of generality, we set $\varepsilon=1$. Then

$A(x)=\{y \in X: x=y$ or $f(x, y) \cap(-w(x, y) e-D) \neq \emptyset\}$,

$\forall x \in X$,

and $S$ is identical to (5). Take $\bar{x}=\widehat{x}$ if $S(\widehat{x})=\emptyset$. Otherwise, there exists $\bar{x} \in S(\widehat{x})$ such that $S(\bar{x})=\emptyset$ by Lemma 9(ii). All in all, $A(\bar{x})=\{\bar{x}\}$. The conclusion (i) is true. Also, $y \neq \bar{x}$ is equivalent to $y \notin A(\bar{x})$, and so the conclusion (ii) holds by Lemma 6(ii). If, further, $S(\widehat{x}) \neq \emptyset$, (i) implies that

$$
f(\widehat{x}, \bar{x}) \cap(-\varepsilon w(\widehat{x}, \bar{x}) e-D) \neq \emptyset,
$$

by Lemma 6(ii). 
Corollary 11. If (a5) is replaced by

(a6) $(X, d)$ is compact

in Theorem 10, then the conclusions still hold.

Proof. It is easy to see that $f_{x}(X)$ is compact for each $x \in X$ by (a1), (a4), (a6), and Lemma 1. The rest proof is divided into three steps.

(a) For any $\lambda, \mu \in \mathbb{R}$ with $\lambda<\mu$,

$\lambda e-D \subset \mu e-(\mu-\lambda) e-D \subset \mu e-\operatorname{int} D-D \subset \mu e-\operatorname{int} D$.

(b) For any $u \in Y$, there exists $\lambda_{u} \in \mathbb{R}$ such that $u \notin \lambda_{u} e-D$. In fact, if $u \in \lambda e-D, \forall \lambda \in \mathbb{R}$, then $u \in \lambda e-\operatorname{int} D, \forall \lambda \in \mathbb{R}$ by (a), which implies

$$
\{\lambda e-u: \lambda \in \mathbb{R}\}=\{-\lambda e-u: \lambda \in \mathbb{R}\} \subset \operatorname{int} D .
$$

Since $Y=\cup\{\lambda e-D: \lambda \in \mathbb{R}\}$ by Lemma 2.1 in [30], for each $v \in Y$, there exists $d_{0} \in \operatorname{int} D$ and $\mu \in \mathbb{R}$ such that $-v=\mu e-d_{0}$. Then $v=-\mu e+d_{0}=(-\mu e-u)+d_{0}+u \in u+\operatorname{int} D$. Thus $Y \subset u+$ int $D$ and a contradiction with properness if $D$ arises.

(c) For some $r \in \mathbb{R}, f_{x}(X) \cap(r e-D)=\emptyset$ and (a5) holds. Indeed, for each $u \in f_{x}(X)$, there exists $\lambda_{u} \in \mathbb{R}$ such that $u \notin \lambda_{u} e-D$, and so $N_{u} \cap\left(\lambda_{u} e-D\right)=\emptyset$ for some $N_{u} \in \mathcal{N}(u)$. By the completeness of $f_{x}(X)$, there exists $u_{1}, \ldots, u_{n} \in f_{x}(X)$ such that $f_{x}(X)=\cup\left\{N_{u_{i}}: 1 \leq i \leq n\right\}$. Taking $r=\min \left\{\lambda_{u_{i}}\right.$ : $1 \leq i \leq n\}$, we have $f_{x}(X) \cap($ re $-D)=\emptyset$.

Apparently, we have the following by applying Theorem 10.

\section{Corollary 12. If}

(a7) $f(x, x) \subset-\partial D, \forall x \in X$, where $\partial D$ is the topological bound of $D$

instead of (a3), hold in Theorem 10, then the conclusions are true as well.

Remark 13. Under all the hypotheses in Theorem 10, Corollary 11, or Corollary 12, for each $\varepsilon>0$, there exists $x_{\varepsilon} \in X$ such that

$$
f\left(x_{\varepsilon}, y\right) \cap\left(-\varepsilon w\left(x_{\varepsilon}, y\right) e-\operatorname{int} D\right)=\emptyset, \quad \forall y \in X .
$$

In fact, it is obvious that $f\left(x_{\varepsilon}, y\right) \cap\left(-\varepsilon w\left(x_{\varepsilon}, y\right) e-\operatorname{int} D\right)=$ $\emptyset, \forall y \in X$ with $y \neq x_{\varepsilon}$. For $y=x_{\varepsilon}$, if

$$
f\left(x_{\varepsilon}, x_{\varepsilon}\right) \cap\left(-\varepsilon w\left(x_{\varepsilon}, x_{\varepsilon}\right) e-\operatorname{int} D\right) \neq \emptyset,
$$

then $f\left(x_{\varepsilon}, x_{\varepsilon}\right) \cap(-$ int $D) \neq \emptyset$, which contradicts with (a3) or (a7).

Remark 14. (a) The condition:

(a8) there exists $\tilde{b} \in Y$ such that $f_{x}(X) \cap(\tilde{b}-\operatorname{int} D)=\emptyset$,

often appeared in the results on Ekeland variational principle for (generalized) vector equilibrium problems, such as Theorem 2.1 in [22]. Actually, (a8) is equivalent to (a5). As the case stands, it is apparent that (a5) implies (a8). On the other hand, taking $b \in \widetilde{b}-\operatorname{int} D$, then $b-D \subset \widetilde{b}-\operatorname{int} D-D \subset \widetilde{b}-\operatorname{int} D$, which guarantees (a5).

(b) The condition:

(a9) for each $x \in X, 0_{Y} \in f(x, x)$ or $f(x, x)=\left\{0_{Y}\right\}$,

instead of (a3) or (a7), is always required to prove variational principle for equilibrium problems in many literatures, such as in [20-24]. But this condition is unnecessary in Theorem 10. See the following examples.

Example 15. Let $X=[0,1], Y=\mathbb{R}^{2}$, and $D=\{(x, y): x \in$ $\mathbb{R}, y \geq 0\}$, and let $d(x, y)=|x-y|, \forall x, y \in X$. Define $f$ : $X \times X \rightarrow 2^{Y}$ as

$$
f(x, y)=\{(x,|y-x|),(-x,|y-x|)\}, \quad \forall x, y \in X .
$$

By computing simply, (a1)-(a5) hold. But (a9) is false since $0_{Y} \notin f(x, x)$ unless $x=0$.

Example 16. Let $X=\mathbb{R}, Y=\mathbb{R}^{2}$, and $D=\{(x, y): x \in \mathbb{R}, y \geq$ $0\}$, and let $d(x, y)=|x-y|, \forall x, y \in X$, and $f: X \times X \rightarrow 2^{Y}$ define as $f(x, y)=[1,2] \times\{0\}, \forall x, y \in X$. It is clear that (a1)(a2), (a4)-(a5), and (a7) are satisfied, but (a9) does not hold.

It is effortless to obtain the following variant of vectorial form of Ekeland variational principle by Theorem 10 .

Theorem 17. Let $\varepsilon, \lambda>0$ be given. If, besides (a1)-(a5),

(a10) for some $\widehat{x} \in X$ with $S(\widehat{x}) \neq \emptyset$,

$$
f(\hat{x}, y) \cap(-\varepsilon e-D)=\emptyset, \quad \forall y \in X,
$$

where $S$ is defined as (26), then there exists $\bar{x} \in X$ such that

(i) $f(\bar{x}, \widehat{x}) \subset D$ if, further, $f(x, x) \subset D, \forall x \in X$;

(ii) $w(\widehat{x}, \bar{x}) \leq \lambda$;

(iii) $f(\bar{x}, y) \cap(-(\varepsilon / \lambda) w(\bar{x}, y) e-D)=\emptyset, \forall y \in X$ with $y \neq \bar{x}$.

Proof. Let $\bar{x} \in X$ be the point provided by Theorem 10 with $\varepsilon / \lambda$ instead of $\varepsilon$. Then (iii) is obtained by the conclusion (ii) in Theorem 10. In addition, in view of the conclusion (i) in Theorem 10,

$$
\zeta_{f_{\widehat{x}}}(\bar{x})+\frac{\varepsilon}{\lambda} w(\widehat{x}, \bar{x}) \leq 0 .
$$

It follows from (34)-(35), (a10), and Lemma 6(ii) that $-\varepsilon \leq$ $\zeta_{f_{\hat{x}}}(\bar{x}) \leq-(\varepsilon / \lambda) w(\hat{x}, \bar{x})$ and so the conclusion (ii) holds. Moreover, according to the conclusion (iii) in Theorem 10, there exists $\tilde{u} \in f(\widehat{x}, \bar{x})$ such that

$$
-\tilde{u} \in \frac{\varepsilon}{\lambda} w(\widehat{x}, \bar{x})+D .
$$

Taking $x=z=\bar{x}$ and $y=\widehat{x}$ in (a2), we have

$$
f(\bar{x}, \widehat{x})+f(\widehat{x}, \bar{x}) \subset f(\bar{x}, \bar{x})+D \subset D .
$$

Then

$$
f(\bar{x}, \widehat{x}) \subset-\tilde{u}+D \subset \frac{\varepsilon}{\lambda} w(\widehat{x}, \bar{x})+D+D \subset D .
$$

Thereby, (i) holds. 
Remark 18. (a) If the solution set of (GVEP2) or (GVEP1) is nonempty, then there must exist a point $\widehat{x} \in X$ satisfying (34).

(b) If, further, $D$ is a point cone (that is, $D \cap(-D)=\left\{0_{Y}\right\}$ ) in Theorems 17, then the condition (a10) can be weakened as follows: for some $\widehat{x} \in X$ with $S(\widehat{x}) \neq \emptyset$,

$$
f(\widehat{x}, x) \cap\left(-\varepsilon e-D \backslash\left\{0_{Y}\right\}\right)=\emptyset, \quad \forall x \in X,
$$

where $S$ is defined as (5).

In fact, we only prove (ii) by the proof of Theorem 17. In view of Theorem 10 (iii),

$$
f(\widehat{x}, \bar{x}) \cap\left(-\frac{\varepsilon}{\lambda} w(\widehat{x}, \bar{x}) e-D\right) \neq \emptyset .
$$

Then there exist $y_{0} \in f(\hat{x}, \bar{x})$ and $d_{0} \in D$ such that $y_{0}=$ $-(\varepsilon / \lambda) w(\hat{x}, \bar{x}) e-d_{0}$. By (39),

$$
-\frac{\varepsilon}{\lambda} w(\widehat{x}, \bar{x}) e-d_{0} \notin-\varepsilon e-D \backslash\left\{0_{Y}\right\},
$$

or equivalently,

$$
\frac{\varepsilon}{\lambda}(w(\widehat{x}, \bar{x})-\lambda) e+d_{0} \notin D \backslash\left\{0_{Y}\right\} .
$$

If $w(\hat{x}, \bar{x})>\lambda$, then $(\varepsilon / \lambda)(w(\hat{x}, \bar{x})-\lambda) e+d_{0} \in D$ and so $(\varepsilon / \lambda)(w(\hat{x}, \bar{x})-\lambda) e+d_{0}=0_{Y}$, which implies that $d_{0}=$ $-(\varepsilon / \lambda)(w(\widehat{x}, \bar{x})-\lambda) e \in-\operatorname{int} D$. Thereupon, $d_{0}=0_{Y}$ by the pointedness of $D$, which contradicts with $d_{0} \in-$ int $D$.

\section{Applications of Vectorial Form of Ekelend Variational Principle for (GVEP1)}

In this section, some existence results of solutions of (GVEP2) are established as applications of Ekelend variational principle for (GVEP1).

Theorem 19. Let $X$ admit a topology $\tau$ (possibly different from the initial topology $\mathscr{T}$ induced by $d$ ). Besides (a1)-(a2), (a4)(a5) and (a7) in the sense of $\mathscr{T}$, if the following conditions hold:

(b1) $X$ is $\tau$-compact; namely, $X$ is compact with respect to $\tau$;

(b2) for each $x \in X, x_{k} \stackrel{\tau}{\rightarrow} x$ as $k \rightarrow+\infty$ implies the boundedness of $\left\{d\left(x_{k}, x\right): k \in \mathbb{N}\right\}$;

(b3) $f^{y}$ is $\tau$-lower semicontinuous on $X$ for each $y \in X$, then (GVEP2) has a solution.

Proof. If $w=d$ and $\varepsilon=1 / k$ in (31), then there exists $x_{k} \in X$ such that

$$
f\left(x_{k}, y\right) \cap\left(-\frac{1}{k} d\left(x_{k}, y\right) e-\operatorname{int} D\right)=\emptyset, \quad \forall y \in X .
$$

Thus $\zeta_{f^{y}}\left(x_{k}\right) \geq-(1 / k) d\left(x_{k}, y\right)$ by Lemma 6(i). Without loss of generality, let $x_{k} \stackrel{\tau}{\rightarrow} x$ by (b1). Since $\zeta_{f^{y}}(\cdot)$ is $\tau$-upper semicontinuous for each $y \in X$ by (b3) and Lemma 7(i),

$$
\begin{aligned}
\zeta_{f^{y}}(\bar{x}) & \geq \limsup _{k \rightarrow+\infty} \zeta_{f^{y}}\left(x_{k}\right) \\
& \geq \limsup _{k \rightarrow+\infty}-\frac{1}{k} d\left(x_{k}, y\right)=0, \quad \forall y \in X,
\end{aligned}
$$

due to (b2). This deduces that $f(\bar{x}, y) \cap(-\operatorname{int} D)=\emptyset, \forall y \in X$ by Lemma 6(i); that is, (GVEP2) has a solution.

Remark 20. It is worth noting that the condition (b2) cannot be guaranteed by (b1). For instance, let $X=\mathbb{R}$ and $d(x, y)=$ $|x-y|, \forall x, y \in X$. For the trivial topology $\tau=\{\emptyset, X\}, X$ is $\tau$-compact. For $x_{k}=k$, it is clear that $x_{k} \stackrel{\tau}{\rightarrow} 0$, but $\left\{d\left(x_{k}, 0\right)\right.$ : $k \in \mathbb{N}\}$ is unbounded.

Corollary 21. If (a1)-(a2), (a4), and (a6)-(a7) and

(a11) $f^{y}$ is lower semicontinuous on $X$ for each $y \in X$,

hold, then (GVEP2) has a solution.

Proof. Clearly, (a6) implies (a5). Taking $\tau=\mathscr{T}$ in Theorem 19, (b1) and (b3) hold by (a6) and (a11), respectively, and (b2) holds trivially since $d\left(x_{k}, \bar{x}\right) \rightarrow 0$ when $x_{k} \rightarrow \bar{x}$. Thus this conclusion is true by Theorem 19 .

When the existence of solutions of equilibrium problems on a noncompact domain is discussed, some sufficient assumptions, such as coercivity condition [31] and condition $C_{1}\left(x_{0}\right)$ [20], and so forth, must be required to be substitute for compact domain. Motivated by these ideas, we obtain the following existence results of solutions of (GVEP2) by applying Theorem 19.

Theorem 22. Let (a1)-(a2), (a4)-(a5), and (a7) hold in the sense of $\mathscr{T}$ induced by $d$, and let $X$ admit a topological $\tau$ (possibly different from the initial topology $\mathscr{T}$ induced by $d$ ). If (b3) is satisfied and the following hold:

(b4) each $\mathscr{T}$-closed bounded ball on $X$ is $\tau$-compact,

(b5) for given point $\hat{x} \in X$, there exists a $\tau$-compact set $C \subset$ $X$ satisfying that, for each $x \in C$ and $\left\{x_{k}\right\} \subset C, x_{k} \stackrel{\tau}{\rightarrow} x$ implies the boundedness of $\left\{d\left(x_{k}, x\right): k \in \mathbb{N}\right\}$ and for each $x \in X \backslash C$,

$d(y, \widehat{x})<d(x, \widehat{x}), \quad f(x, y) \cap(-D) \neq \emptyset$

for some $y \in X$,

(b6) for each $x \in X, L(x)=\{y \in X: f(x, y) \cap(-D) \neq \emptyset\}$ is $\tau$-closed,

(b7) for each $y \in X, d(\cdot, y)$ is $\tau$-lower semicontinuous, then (GVEP2) has a solution.

Proof. Define $A: X \rightarrow 2^{Y}$ as

$$
A(x)=\left\{y \in X: d(y, \widehat{x}) \leq d(x, \widehat{x}), \zeta_{f_{x}}(y) \leq 0\right\} .
$$

We see the following.

(a) For each $x \in X, A(x) \neq \emptyset$ since $x \in A(x)$ by (a7).

(b) For each $x \in X, y \in A(x)$ implies that $A(y) \subset A(x)$ by the similar argument of the proof in Lemma 9.

(c) $A(x)$ is $\tau$-compact for each $x \in X$ by (b4) and (b6). 
By regarding the $\tau$-compact set $C$ as $X$ in Theorem 19, there exists $\bar{x}_{C} \in C$ such that

$$
f\left(\bar{x}_{C}, y\right) \cap(-\operatorname{int} D)=\emptyset, \quad \forall y \in C .
$$

Now argue the conclusion by contradiction. Assume that there exists $\bar{y} \in X \backslash C$ such that

$$
f\left(\bar{x}_{C}, \bar{y}\right) \cap(-\operatorname{int} D) \neq \emptyset .
$$

Let

$$
\alpha=\min \{d(y, \widehat{\mathrm{x}}): y \in A(\bar{y})\}
$$

Now assert that

$$
A(\bar{y}) \cap C=\emptyset .
$$

In fact, if $y \in A(\bar{y}) \cap C$, then $f(\bar{y}, y) \cap(-D) \neq \emptyset$, which, together with (48), deduces that $f\left(\bar{x}_{C}, y\right) \cap(-$ int $D) \neq \emptyset$ in view of (a2) and Lemmas 6 and 8. This contradicts with (47).

The minimum in (49) is attained since $A(\bar{y})$ is nonempty $\tau$-compact by (a) and (c), and $d(\cdot, \widehat{x})$ is $\tau$-lower semicontinuous. Take $z_{0} \in A(\bar{y})$ such that $d\left(z_{0}, \hat{x}\right)=\alpha$. Since $z_{0} \notin C$ by (50), $z_{1}$ can be chosen to satisfy that $d\left(z_{1}, \widehat{x}\right)<d\left(z_{0}, \widehat{x}\right)=\alpha$ and $f\left(z_{0}, z_{1}\right) \cap(-D) \neq \emptyset$ by (b5). Accordingly, the assertion $z_{1} \in A\left(z_{0}\right) \subset A(\bar{y})$ is absurd by the definition of $\alpha$. Thereby, $f\left(\bar{x}_{C}, y\right) \cap(-\operatorname{int} D)=\emptyset, \forall y \in X$, or in other words, $\bar{x}_{C}$ is a solution of (GVEP2).

Theorem 23. Suppose that $(X,\|\cdot\|)$ is a real reflexive Banach space. Besides (a1)-(a2), (a4)-(a5) and (a7), if the following conditions hold:

(b8) for each $y \in X, f^{y}$ is weakly lower semicontinuous,

(b9) there exists $r>0$ such that for each $x \in X \backslash X_{r}, y \in$ $X$ can be selected such that $\|y\|<\|x\|$ and $f(x, y) \cap$ $(-D) \neq \emptyset$, where $X_{r}=\{x \in X:\|x\| \leq r\}$,

then (GVEP2) has a solution.

Proof. Denote $\mathscr{T}$ and $\tau$ by the strong topology and the weak topology on $X$, respectively, and define $A: X \rightarrow 2^{X}$ as

$$
A(x)=\left\{y \in X:\|y\| \leq\|x\|, \zeta_{f_{x}}(y) \leq 0\right\}, \quad \forall x \in X .
$$

Letting $\widehat{x}=0_{X}, C=X_{r}$, and $d(x, y)=\|x-y\|, \forall x, y \in X$, we see that $A(x)$ is just equal to (46). (b3) and (b5) are satisfied by (b8) and (b9), respectively. Also, (b4) and (b7) hold thanks to weak compactness of each closed bounded ball and weak lower semicontinuity of $\|\cdot\|$ in a real reflexive Banach space, respectively. Besides, (b6) guarantees the weak compactness of $A(x)$ in the proof of Theorem 22. While this property can be guaranteed by the assertions that $X_{\|x\|}$ is weakly compact and $\left\{y \in X: \zeta_{f_{x}}(y) \leq 0\right\}$ is closed by (a4) and Lemma 7(ii), this rest proof completes by the similar argument of the proof in Theorem 22.

\section{Several Equivalent Results of Vectorial Form of Ekelend Variational Principle}

In this section, we shall present several equivalent results of the established Ekelend variational principle. Now Define $\preceq$ as follows:

$$
x \preceq y \Longleftrightarrow x=y \quad \text { or } \quad f(x, y) \cap(-w(x, y) e-D) \neq \emptyset .
$$

Then, under the conditions (a1)-(a3), $\preceq$ is a quasiorder on $X$ by Lemma $8(\mathrm{i})$. Also, $(X, \preceq)$ has a maximal element $\bar{x}$ under all assumptions of Theorem 10. Indeed, $A(x)=\{y \in X: x \preceq$ $y\}$, where $A(x)$ is defined as (27). Then there exists $\bar{x} \in X$ such that $A(\bar{x})=\{\bar{x}\}$ by Theorem $10(\mathrm{i})$, which implies that $\bar{x}$ is a maximal element of $(X, \preceq)$.

Theorem 24. Let (a1)-(a3) hold and X equip a quasiorder $\preceq$ defined as (52). Then the following assertions are equivalent.

(i) (Existence result of maximal element) $(X, \preceq)$ has a maximal element $\bar{x}$.

(ii) (Ekelend variational principle for (GVEP1)) There exists $\bar{x} \in X$ such that

$$
f(\bar{x}, y) \cap(-w(\bar{x}, y) e-D)=\emptyset, \quad \forall y \in X \text { with } y \neq \bar{x} .
$$

(iii) (Caristi-kirk fixe-point theorem) Let $P: X \rightarrow 2^{X}$ be a strict, set-valued mapping such that, for any $x \in X, x \preceq$ $y, \forall y \in P(x)$. Then there exists $\bar{x} \in X$ such that $P(\bar{x})=$ $\{\bar{x}\}$.

Proof. (i) $\Rightarrow$ (iii) Let $\bar{x}$ be a maximal element on $(X, \preceq)$. If $P(\bar{x}) \neq\{\bar{x}\}$, then there exists $\tilde{y} \neq \bar{x}$ such that $\tilde{y} \in P(\bar{x})$ since $P$ is strict. By the assumption (iii), $\bar{x} \preceq \tilde{y}$, which is absurd. Thus $P(\bar{x})=\{\bar{x}\}$.

(iii) $\Rightarrow$ (ii) Let $P=A$, where $A(x)$ is defined as (27). Then for any $x \in X, x \preceq y, \forall y \in P(x)$; that is, the assumption of (iii) is satisfied. Consequently, $P(\bar{x})=\{\bar{x}\}$ for some $\bar{x} \in X$ and so (ii) holds.

(ii) $\Rightarrow$ (i) Define $A(x)$ as (27). Then $A(x)=\{y \in X: x \preceq$ $y$, and there exists $\bar{x} \in X$ such that $A(\bar{x})=\{\bar{x}\}$ by (ii). This implies that $\bar{x}$ is a maximal element on $(X, \preceq)$.

Theorem 25. Let $S: X \rightarrow 2^{X}$ be defined as (5) and $\hat{x} \in$ $X$ be any given point with $S(\widehat{x}) \neq \emptyset$. Then the results below are equivalent.

(i) (Ekelend variational principle for (GVEP1)) There exists $\bar{x} \in S(\widehat{x})$ such that (53) holds.

(ii) (Existence result of solutions of $(G V E P 1))$ If, for any $x \in$ $S(\widehat{x})$,

$$
f(x, y) \cap(-D) \neq \emptyset \quad \text { for some } y \in X
$$

implies that there exists $y \in X$ with $y \neq x$ such that

$$
f(x, y) \cap(-w(x, y) e-D) \neq \emptyset,
$$

then (GVEP1) has a solution $\bar{x} \in S(\widehat{x})$. 
(iii) (Caristi-kirk type fixe-point Theorem) Let $P: X \rightarrow$ $2^{X}$ be a strict, set-valued mapping such that, for any $x \in S(\widehat{x})$, there exists $y \in P(x)$ satisfying (54). Then there exists $\bar{x} \in S(\widehat{x})$ such that $\bar{x} \in P(\bar{x})$.

(iv) (Oettli-Théra type Theorem) Let $\Phi \subset X$. If, for every $x \in S(\widehat{x}) \backslash \Phi$, there exists $y \in X$ with $y \neq x$ such that (54) holds, then $S(\widehat{x}) \cap \Phi \neq \emptyset$.

Proof. (i) $\Rightarrow$ (iv) Let $\bar{x} \in S(\widehat{x})$ satisfy (53). If $\bar{x} \notin \Phi$, then there exists $y \in X$ with $y \neq x$ such that $f(\bar{x}, y) \cap(-w(\bar{x}, y) e-D) \neq \emptyset$ holds by the hypothesis of (iv). This is absurd by (53) and so $\bar{x} \in S(\widehat{x}) \cap \Phi$.

(iv) $\Rightarrow$ (i) Let (iv) hold and define $\Phi=\{x \in X: S(x)=\emptyset\}$. If $x \notin \Phi$, then $S(x) \neq \emptyset$; that is, there exists $y \in X$ with $y \neq x$ such that (54) holds; that is, the assumption of (iv) is satisfied. Thus, $S(\widehat{x}) \cap \Phi \neq \emptyset$. By taking $\bar{x} \in S(\widehat{x}) \cap \Phi, \bar{x}$ satisfies (53); that is, (i) is true.

(ii) $\Rightarrow$ (iv) This conclusion is argued by contradiction. Assume that $S(\widehat{x}) \cap \Phi=\emptyset$. If $x \in S(\widehat{x})$, then $x \notin \Phi$ and so there exists $y \in X$ with $y \neq x$ such that (54) holds by the hypothesis of (iv). Thus the assumption (ii) is satisfied and so there exists $\bar{x} \in S(\widehat{x})$ such that

$$
f(\bar{x}, y) \cap(-D)=\emptyset, \quad \forall y \in X,
$$

which implies that

$$
f(\bar{x}, y) \cap(-w(\bar{x}, y) e-D)=\emptyset, \quad \forall y \in X
$$

by $w(\bar{x}, y) \geq 0$ and $e \in \operatorname{int} D$. This contradicts with (54).

(iv) $\Rightarrow$ (ii) Let $\Phi=\{x \in X: f(x, y) \cap(-D)=\emptyset, \forall y \in X\}$. By the assumption of (ii), for any $x \in S(\widehat{x}) \backslash \Phi$, there exists $y \in$ $X$ with $y \neq x$ such that (54) holds. This is just the assumption of (iv). Choosing $\bar{x} \in S(\widehat{x}) \cap \Phi$, we have that $\bar{x} \in S(\widehat{x})$ and $f(\bar{x}, y) \cap(-D)=\emptyset, \forall y \in X$.

(iii) $\Rightarrow$ (iv) Define $P: X \rightarrow 2^{X}$ as $P(x)=\{y \in X:$ $y \neq x\}$. Argue this by contradiction. If $S(\widehat{x}) \cap \Phi=\emptyset$, then the assumption of (iii) holds by the assumption of (iv). Hence, $P$ has a fixed point on $X$, which is absurd by the definition of $P$.

(iv) $\Rightarrow$ (iii) Let $\Phi=\{x \in X: x \in P(x)\}$. The assumption of (iv) is deduced straight by the assumption of (iii). Resultingly, $S(\widehat{x}) \cap \Phi \neq \emptyset$; that is, $\bar{x} \in P(\bar{x})$ for some $\bar{x} \in S(\widehat{x})$.

Remark 26. Suppose that $f$ is a single-valued mapping. Theorem 25 reduces Theorem 5.1 in [21] if $f(x, x)=0_{Y}, \forall x \in$ $X$ hold instead of (a3), and if, further, (a4) hold and $f_{x}$ is $(e, D)$-lower semicontinuous for each $x \in X$ (namely, $\{y \in$ $X: f(x, y) \in r e-D\}$ is closed for each $r \in \mathbb{R}$ and each $x \in X)$.

\section{Acknowledgments}

This research was supported by the Doctoral Fund of Innovation of Beijing University of Technology; the Science and Technology Development Project of Siping (2011005).

\section{References}

[1] I. Ekeland, "Sur les problèmes variationnels," Comptes Rendus de l'Académie des Sciences, vol. 275, pp. A1057-A1059, 1972.
[2] I. Ekeland, "On the variational principle," Journal of Mathematical Analysis and Applications, vol. 47, pp. 324-353, 1974.

[3] I. Ekeland, "Nonconvex minimization problems," American Mathematical Society. Bulletin, vol. 1, no. 3, pp. 443-474, 1979.

[4] J. P. Aubin and I. Ekeland, Applied Nonlinear Analysis, John Wiley \& Sons, New York, NY, USA, 1984.

[5] G. Y. Chen and X. X. Huang, "Ekeland's $\epsilon$-variational principle for set-valued mappings," Mathematical Methods of Operations Research, vol. 48, no. 2, pp. 181-186, 1998.

[6] A. Göpfert, H. Riahi, C. Tammer, and C. Zălinescu, Variational Methods in Partially Ordered Spaces, vol. 17 of CMS Books in Mathematics, Springer, New York, NY, USA, 2003.

[7] T. X. D. Ha, "Some variants of the Ekeland variational principle for a set-valued map," Journal of Optimization Theory and Applications, vol. 124, no. 1, pp. 187-206, 2005.

[8] L. J. Lin and W. S. Du, "Ekeland's variational principle, minimax theorems and existence of nonconvex equilibria in complete metric spaces," Journal of Mathematical Analysis and Applications, vol. 323, no. 1, pp. 360-370, 2006.

[9] C. Gutiérrez, B. Jiménez, and V. Novo, "A set-valued Ekeland's variational principle in vector optimization," SIAM Journal on Control and Optimization, vol. 47, no. 2, pp. 883-903, 2008.

[10] P. Q. Khanh and D. N. Quy, “On generalized Ekeland's variational principle and equivalent formulations for set-valued mappings," Journal of Global Optimization, vol. 49, no. 3, pp. 381-396, 2011.

[11] P. Q. Khanh and D. N. Quy, "Versions of Ekeland's variational principle involving set perturbations," Journal of Global Optimization, 2012.

[12] J. P. Aubin, Mathematical Methods of Game and Economic Theory, North-Holland, Amsterdam, The Netherlands, 1979.

[13] I. Ekeland, "Some lemmas about dynamical systems," Mathematica Scandinavica, vol. 52, no. 2, pp. 262-268, 1983.

[14] J. T. Xing, “A note about Oden's constitutive variational principle," Applied Mathematics and Mechanics, vol. 8, no. 10, pp. 975984, 1987.

[15] G. Isac, "Nuclear cones in product spaces, Pareto efficiency and Ekeland-type variational principles in locally convex spaces," Optimization, vol. 53, no. 3, pp. 253-268, 2004.

[16] E. Blum and W. Oettli, "From optimization and variational inequalities to equilibrium problems," The Mathematics Student, vol. 63, no. 1-4, pp. 123-145, 1994.

[17] S. Al-Homidan, Q. H. Ansari, and J. C. Yao, "Some generalizations of Ekeland-type variational principle with applications to equilibrium problems and fixed point theory," Nonlinear Analysis. Theory, Methods \& Applications A, vol. 69, no. 1, pp. 126-139, 2008.

[18] K. R. Kazmi, "A variational principle for vector equilibrium problems," Indian Academy of Sciences. Proceedings. Mathematical Sciences, vol. 111, no. 4, pp. 465-470, 2001.

[19] Q. H. Ansari, I. V. Konnov, and J. C. Yao, "Existence of a solution and variational principles for vector equilibrium problems," Journal of Optimization Theory and Applications, vol. 110, no. 3, pp. 481-492, 2001.

[20] M. Bianchi, G. Kassay, and R. Pini, "Ekeland's principle for vector equilibrium problems," Nonlinear Analysis. Theory, Methods \& Applications A, vol. 66, no. 7, pp. 1454-1464, 2007.

[21] Q. H. Ansari, "Vectorial form of Ekeland-type variational principle with applications to vector equilibrium problems and fixed point theory," Journal of Mathematical Analysis and Applications, vol. 334, no. 1, pp. 561-575, 2007. 
[22] Y. Araya, K. Kimura, and T. Tanaka, "Existence of vector equilibria via Ekeland's variational principle," Taiwanese Journal of Mathematics, vol. 12, no. 8, pp. 1991-2000, 2008.

[23] S. Eshghinezhad and M. Fakhar, "Vectorial form of Ekelandtype variational principle in locally convex spaces and its applications," Fixed Point Theory and Applications, vol. 2010, Article ID 276294, 15 pages, 2010.

[24] J. Zeng and S. J. Li, "An Ekeland's variational principle for setvalued mappings with applications," Journal of Computational and Applied Mathematics, vol. 230, no. 2, pp. 477-484, 2009.

[25] D. T. Luc, Theory of Vector Optimization, vol. 319 of Lecture Notes in Economics and Mathematical Systems, Springer, Berlin, Germany, 1989.

[26] J. P. Aubin and A. Cellina, Differential Inclusion, Springer, Berlin, Germany, 1994.

[27] O. Kada, T. Suzuki, and W. Takahashi, "Nonconvex minimization theorems and fixed point theorems in complete metric spaces," Mathematica Japonica, vol. 44, no. 2, pp. 381-391, 1996.

[28] P. H. Sach and L. A. Tuan, "New scalarizing approach to the stability analysis in parametric generalized Ky Fan inequality problems," Journal of Optimization Theory and Applications, vol. 157, no. 2, pp. 347-364, 2013.

[29] L. A. Tuan, G. M. Lee, and P. H. Sach, "Upper semicontinuity in a parametric general variational problem and application," Nonlinear Analysis. Theory, Methods \& Applications A, vol. 72, no. 3-4, pp. 1500-1513, 2010.

[30] G. Y. Chen and X. Q. Yang, "Characterizations of variable domination structures via nonlinear scalarization," Journal of Optimization Theory and Applications, vol. 112, no. 1, pp. 97-110, 2002.

[31] M. Bianchi and R. Pini, "Coercivity conditions for equilibrium problems," Journal of Optimization Theory and Applications, vol. 124, no. 1, pp. 79-92, 2005. 


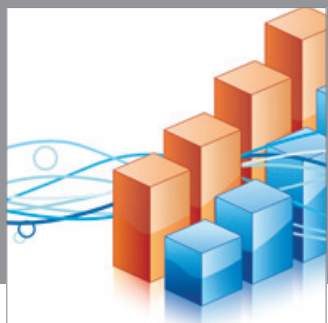

Advances in

Operations Research

mansans

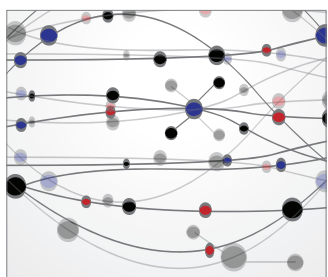

The Scientific World Journal
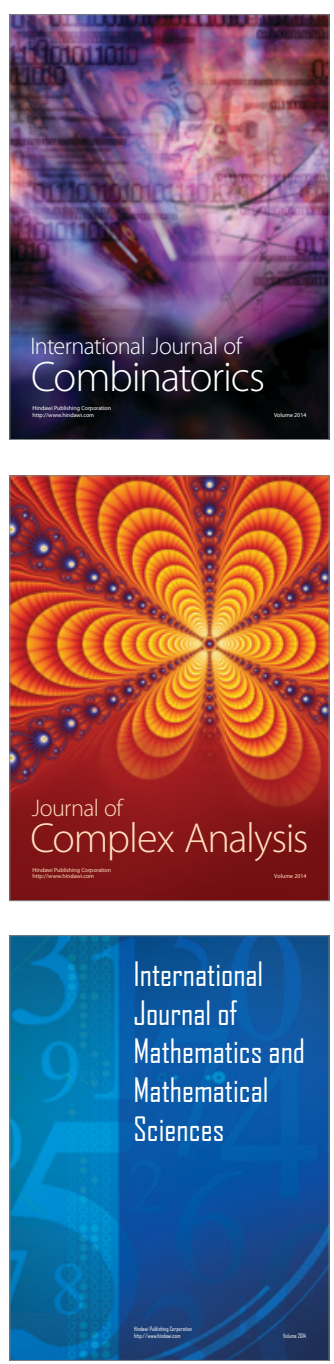
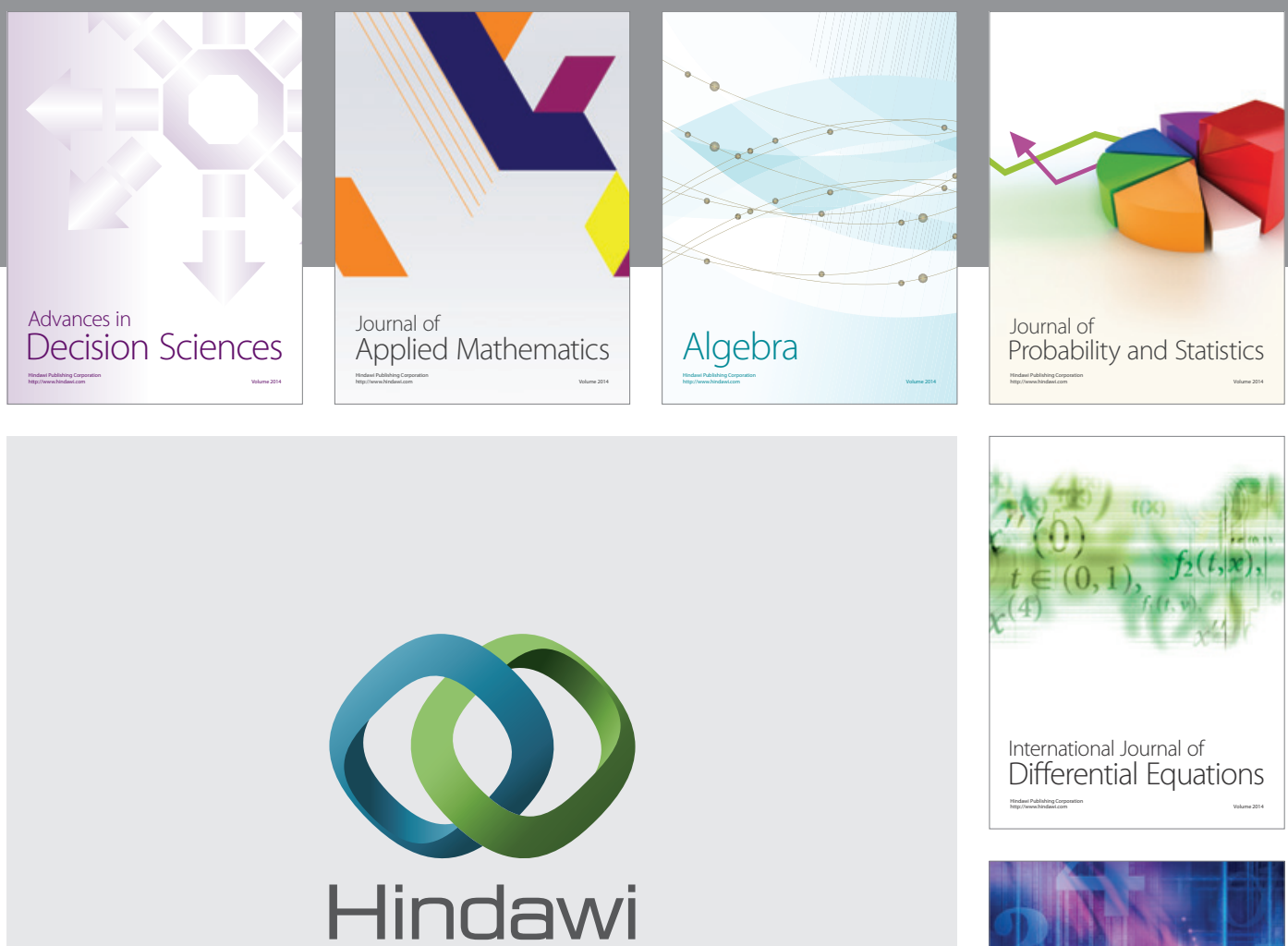

Submit your manuscripts at http://www.hindawi.com
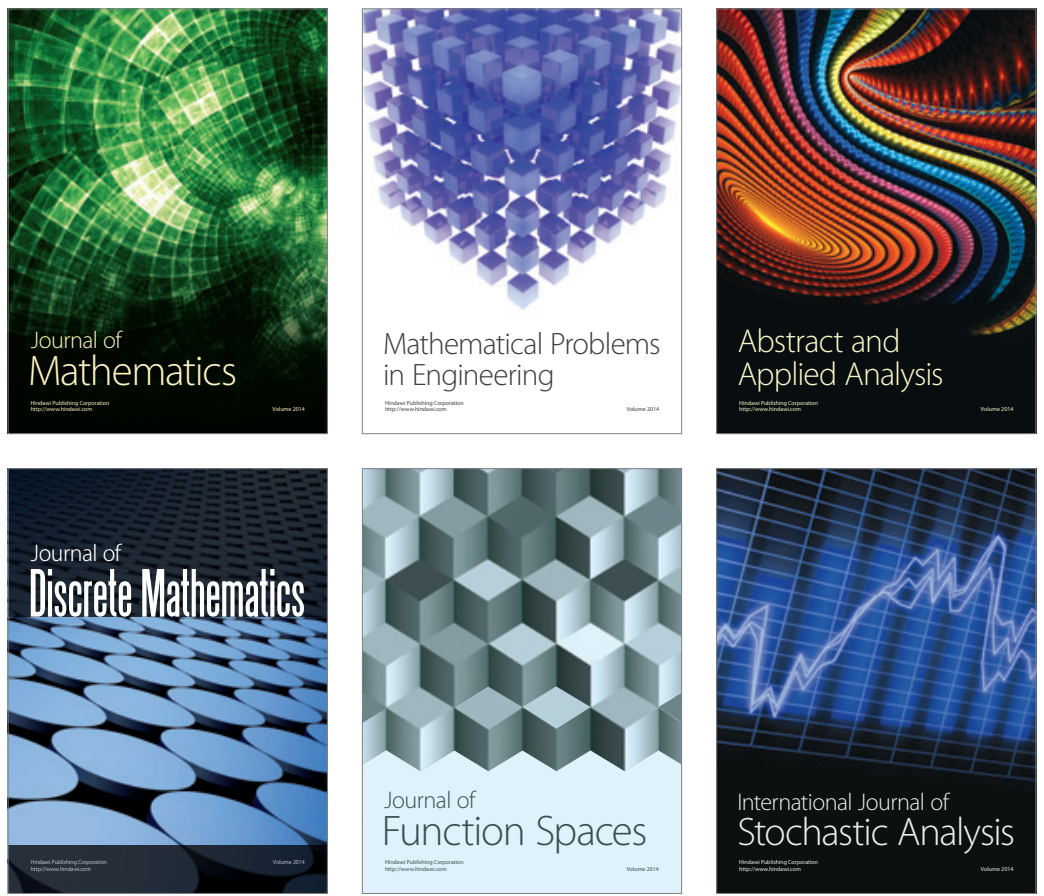

Journal of

Function Spaces

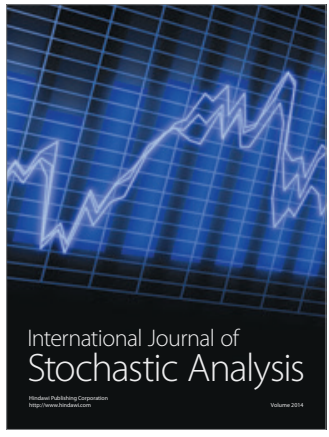

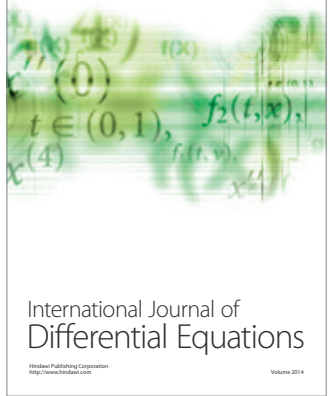
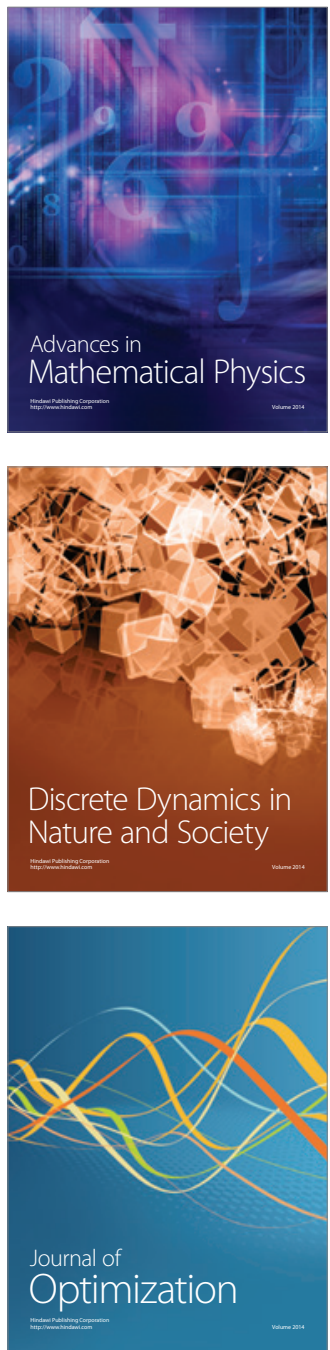